\title{
Los Materiales Didácticos en las Escuelas de Hospital: un Proyecto de Aprendizaje-Servicio para Atender a la Diversidad del Alumnado HOSPITALIZADO $^{1}$
}

\author{
Materiais DidÁticos em Escolas de Hospital: um PRojeto de APRENdizagem \\ DE SERVIÇOS para ATENDER À Diversidade DE ALUNos Hospitalizados
}

\author{
Yésica Teijeiro $\mathrm{BOO}^{2}$ \\ Jesús Rodríguez RODRÍGUEZ ${ }^{3}$ \\ Antía Cores TORRES ${ }^{4}$
}

\begin{abstract}
RESUMEN: Apoyando una educación inclusiva que garantice el derecho a la educación de todas las personas, sea cual sea su situación particular, en este artículo se presentan los resultados de un proyecto de Aprendizaje-Servicio universitario centrado en el desarrollo de una propuesta de materiales didácticos elaborados para atender las necesidades de apoyo educativo específicas del alumnado en situación de enfermedad de una escuela de hospital. Los materiales didácticos han sido elaborados conjuntamente por profesorado y alumnado de las materias de Pedagogía Hospitalaria y Diseño y Evaluación de Materiales Didácticos del 4 o curso del Grado de Pedagogía. Igualmente se contó en el proceso con la colaboración de profesionales del hospital. De este modo, se mejoran las condiciones y los recursos disponibles para la intervención educativa con los pacientes pediátricos durante su ingreso hospitalario, destacando como principales conclusiones del proyecto el alto grado de satisfacción del alumnado universitario participante, ya que tuvieron la oportunidad de poner en práctica en un contexto real los aprendizajes adquiridos en las clases, además de hacer un servicio a la comunidad educativa en los hospitales cubriendo la necesidad de elaborar materiales didácticos específicos para las escuelas hospitalarias. Además, con la participación en esta experiencia tuvieron la oportunidad de trabajar profesionalmente en un equipo interdisciplinar. Todo ello, sin olvidar que los resultados aportan también beneficios directamente para el alumnado del aula hospitalaria con la que se colabora en el proyecto, ya que se amplían los recursos y materiales educativos disponibles en este espacio.
\end{abstract}

PALABRAS CLAVE: Aulas hospitalarias. Materiales didácticos. Aprendizaje-servicio. Docencia universitaria.

RESUMO: Apoiando uma educaçấo inclusiva que garanta o direito à educaçáo de todas as pessoas, em qualquer situaçâo particular, este artigo apresenta os resultados de um projeto Serviço-Ensino Universitário voltado para o desenvolvimento de uma proposta de materiais didáticos elaborados para atender às necessidades específicas de apoio educacional de alunos doentes em uma escola de hospital. Os materiais didáticos foram elaborados em conjunto por professores e alunos das disciplinas de Pedagogia Hospitalar e Desenho e Avaliaçáo de Materiais Didáticos do $4^{\circ}$ ano da Licenciatura em Pedagogia. O processo também contou com a colaboração de profissionais do hospital. Dessa forma, melhoram-se as condiçôes e os recursos disponíveis para intervenção educativa junto ao doente pediátrico durante a sua internação, destacando, como principais conclusôes, o elevado grau de satisfaçáo dos universitários, por terem a oportunidade de concretizar, em um contexto real, a aprendizagem adquirida nas aulas, além de atuarem profissionalmente em uma equipe interdisciplinar, além de prestarem um serviço à comunidade educacional em hospitais, atendendo à necessidade de desenvolvimento de materiais didáticos específicos para escolas hospitalares. Além disso, por participarem dessa experiência, tiveram a oportunidade de atuar profissionalmente em uma equipe interdisciplinar. Tudo isso sem esquecer que os resultados também trazem benefícios diretos para os alunos da sala de aula do hospital com o qual o projeto está colaborando, uma vez que os recursos e os materiais didáticos disponibilizados nesse espaço são ampliados.

PALAVRAS-CHAVE: Escolas de hospital Materiais de ensino. Aprendizado de serviço. Ensino universitário.

\footnotetext{
${ }^{1}$ https://doi.org/10.1590/1980-54702022v28e0094

${ }^{2}$ Profesora Interina de Substitución de la Universidade de Santiago de Compostela. Santiago de Compostela/España. E-mail: yesica.teijeiro@usc.es. ORCID: https://orcid.org/0000-0003-3957-9796

${ }^{3}$ Profesor Titular. Universidad de Santiago de Compostela. Santiago de Compostela/España. E-mail: jesus.rodriguez.rodriguez@ usc.es. ORCID: https://orcid.org/0000-0003-4194-2574

${ }^{4}$ Doctorando en Equidad e Innovación en Educación de la Universidad de Santiago de Compostela. Santiago de Compostela/ Espańa. E-mail: antia.cores.torres@usc.es. ORCID: https://orcid.org/0000-0002-4152-9889
} 


\section{INTRODUCCIÓN}

Según se reconoce en la Declaración Universal de los Derechos Humanos (1948), la educación es un derecho fundamental básico para todos; de ahí que distintas normativas, reglamentos y documentos legales lo contemplen como un deber de obligado cumplimiento. Es por ello, que los distintos Estados se ven obligados a adecuar la oferta formativa a las diversas necesidades y situaciones del alumnado, con programas educativos que atiendan su diversidad y especificidad.

A nivel nacional como internacional existen distintas normativas legales que han ido acompañando el desarrollo de las intervenciones socioeducativas dentro del contexto hospitalario, dirigidas tanto a apoyar la atención al menor en situación de enfermedad, como a garantizar los derechos de la infancia en general, y en particular, para la hospitalizada.

En el escenario internacional, como avance en la protección, definición y promoción de los derechos de los menores, cabe destacar la aprobación por la Asamblea General de las Naciones Unidas, después de varias Declaraciones, de la Convención sobre los Derechos del Niño (1989). Se trata de uno de los tratados internacionales más ratificado a nivel mundial. No es un texto meramente declarativo, sino de una Convención, lo que implica que será necesario desarrollar políticas y recursos por parte de los Estados miembros para que se cumplan los derechos de la infancia (Guilló, 2006). El articulado de esta Convención contempla derechos referidos al desarrollo (art. 6.2), a una atención sanitaria (art. 24), a una educación gratuita (28), a desarrollar plenamente su personalidad y sus habilidades (29), derecho a descansar y a jugar (31), entre otros.

El Informe Warnock, publicado en 1978 y difundido a lo largo de la siguiente década, contempla una serie de recomendaciones que implican un giro importante en la historia de la Educación Especial, reconfigurando la concepción más clásica o tradicional de esta área; así, se presta atención a las necesidades educativas especiales de los sujetos que hay que atender de una forma individualizada, de acuerdo con sus características y en los centros y en las aulas de régimen normal. De este modo se facilita el aprendizaje de los contenidos curriculares, su desarrollo personal e inclusión social. Por otra parte, el Informe Warnock constituye el primer documento que hace referencia a la educación de los niños hospitalizados, lo que comporta la vinculación que se da entre la Pedagogía Hospitalaria y la Educación Especial en esos momentos.

También a nivel internacional cabe mencionar la Resolución del Parlamento Europeo (Diario Oficial de las Comunidades Europeas) en 1986, en la que se aprueba la Carta Europea sobre los Derechos del Niño Hospitalizado.

Con el apoyo de toda la normativa que surge para proteger y amparar a los menores en situación de enfermedad y hospitalización y coincidiendo con una mayor toma en consideración de sus cuidados integrales, se ha incrementado la necesidad de que-mientras el personal sanitario se preocupa de atender la salud física del paciente- existan otra serie de profesionales que cuiden los aspectos psicológicos, educativos y sociales de estos pacientes. En este sentido, uno de sus principales desafíos consistirá en humanizar la estancia del niño, niña o adolescente, actuando preventivamente sobre los posibles efectos negativos que el tratamiento médico y el propio hospital pueden generar (Lizasoáin \& Polaino, 1998). También será básico que los 
recursos y materiales educativos no solo constituyan una herramienta para el entretenimiento, sino que también se conviertan en una herramienta fundamental para el aprendizaje y el juego.

Con estos propósitos ha surgido y se ha ido consolidando la Pedagogía Hospitalaria, convirtiéndose en el marco de las actuaciones socioeducativas que se vienen promoviendo en los contextos hospitalarios, una atención apropiada y una educación inclusiva, que proporcione apoyo y bienestar a la infancia en situación de enfermedad (Grau \& Ortiz, 2001).

Históricamente en la legislación española y otras políticas educativas europeas, la Pedagogía Hospitalaria se relacionó directamente con la Educación Especial, al considerar que en los nińos o nińas hospitalizados, hay necesidades educativas especiales que requieren actuaciones también especiales, debido a múltiples y variadas razones, entre las que destaca la llegada a un lugar desconocido, la separación de la familia, la ruptura con la vida cotidiana, la preocupación por el absentismo escolar, la incertidumbre sobre el pronóstico de la enfermedad o, simplemente, el hecho de sentirse mal (Ortiz, 2001). Sin embargo, no debe obviarse que las necesidades educativas que surgen en este contexto son, en la mayoría de los casos, transitorias: están asociadas a diferentes factores, como pueden ser el motivo de la hospitalización, el contexto hospitalario, la salud del menor, las experiencias previas del paciente y sus familiares, la atención recibida, etc. Aludimos, por tanto, a una educación inclusiva que garantice el derecho a la educación de todas las personas, sea cual sea su situación particular, ya que aun siendo todos diferentes tenemos igualdad de derechos. Por esta razón, la infancia en situación de enfermedad y que requiere hospitalización no tendrá unas condiciones diferentes a las de aquellos que pueden ir a una escuela ordinaria y, por tanto, sin perder su derecho a una educación pública, de calidad y adaptada a sus necesidades, tal y como sostienen las normativas actualmente vigentes.

En este sentido, consideramos que la finalidad de la Pedagogía Hospitalaria es el desarrollo integral de las personas, requiriendo de los profesionales de la educación -entre ellos de los pedagogos- una intervención social y educativa dirigida tanto al paciente pediátrico como a su familia y al contexto que le rodea. Tradicionalmente, la Pedagogía Hospitalaria, se ha aplicado al ámbito infantil, pero sin duda puede y debe extenderse también al de los adultos hospitalizados. Se trata, en definitiva, de la importancia de atender a unas necesidades fundamentales de la persona en situación de enfermedad, algo que rebasa la acción ordinaria del personal sanitario.

\section{El Aprendizaje-Servicio en el Contexto de la Pedagogía Hospitalaria}

En las últimas décadas se ha producido un aumento de las experiencias de Aprendizaje Servicio (en adelante ApS) en contextos universitarios, metodología que siguiendo a Puig Rovira et al. "combina procesos de aprendizaje y servicio a la comunidad en un solo proyecto bien articulado en el que los participantes aprenden a trabajar sobre las necesidades reales del entorno con la finalidad de mejorarlo" (Puig Rovira et al., 2006, p. 22). Estas prácticas están siendo potenciadas desde las propias instituciones diseńando, como es el caso de algunas universidades, convocatorias de innovación educativa por medio del Servicio de Participación e Integración Universitaria, como es el caso de la que presentamos a continuación.

Autores como Rodríguez Gallego (2014) afirman que el ApS en el Espacio Superior de Educación aporta grandes beneficios en tres ámbitos fundamentales: 
- Currículum académico al impulsar una formación práctica relacionada con el posterior desempeño profesional y adaptada al contexto social y económico actual.

- Formación en valores de futuros profesionales que tendrán un papel activo y comprometido en la sociedad.

- Vinculación con la comunidad ya que los proyectos y propuestas surgen de demandas o problemas reales de la sociedad.

Estos tres ámbitos se aglutinan en este proyecto cuyo origen se sitúa en las carencias de materiales didácticos detectadas en el marco de las aulas hospitalarias, con el propósito de contribuir a mejorar la calidad de vida de los pacientes pediátricos desde diferentes ámbitos. La atención educativa en los contextos hospitalarios será lo que promueva la necesidad de implantar actuaciones que reajusten el equilibrio del menor (Latorre \& Blanco, 2010). Estas cuestiones también deben de reflejarse en los materiales didácticos que se emplean para el trabajo socioeducativo que se desarrolla en las aulas hospitalarias.

Sin embargo, los recursos más específicos para este tipo de intervención son muy limitados y se reducen en general a algunos cuentos o juegos en los que se trata el tema de las diferentes enfermedades, a algún recurso creado por fundaciones especializadas en el apoyo de la infancia en situación de enfermedad y hospitalización, o algún material didáctico elaborado por el propio profesorado de las aulas hospitalarias con objetivos específicos para desempeñar en su trabajo. También está en auge el desarrollo de videojuegos que le permiten al paciente pediátrico sentirse fuera del contexto hospitalario e introducirse en un ambiente lúdico y didáctico (Guerra \& Revuelta, 2015).

A pesar de estas iniciativas, la falta de materiales específicos queda patente en el análisis del trabajo diario en estos espacios educativos, tal como se referencia en investigaciones recientes en la que se recomienda:

realizar una revisión, análisis y valoración de aquellos materiales y recursos didácticos que son usados en las aulas hospitalarias de forma diaria y estudiar sus principales características, con el propósito de ver en qué medida responden a las necesidades que plantea el alumnado hospitalizado -en situación de enfermedad-; así como para poder plantear el diseño y elaboración de otros materiales alternativos que cumplan con los objetivos y especificidad que se persiguen desde estos espacios educativos. (Teijeiro, 2019, p. 294)

Por esta razón surge la iniciativa de nuestro proyecto de ApS en el que el alumnado de Pedagogía pudiera contribuir a beneficiar el trabajo de los docentes de las aulas hospitalarias, intentando enriquecer las clases con materiales más contextualizados y personalizados que se conviertan en recursos para realizar aprendizajes con los menores, fomentando la participación y motivación en las clases y, sobre todo, respondiendo a las nuevas necesidades que se plantean en una situación como la que están atravesando en el hospital (Ottaviano \& González, 2015). 


\subsection{La METOdología Y EL DESARrollo DEL PROYECTO "MALETA DE EMERGENCIAS PEDAGÓGICAS: MATERIALES DIDÁCTICOS EN AULAS HOSPITALARIAS”}

En este contexto de escasez de materiales didácticos específicos para trabajar en las aulas hospitalarias surge el proyecto la "Maleta de emergencias pedagógicas: Materiales Didácticos en aulas hospitalarias", iniciado en el curso académico 2018-2019 en la escuela hospitalaria del Complejo Hospitalario Universitario de Ferrol, en el que son participantes directos alumnado de $4^{\circ}$ Grado de Pedagogía de la Universidad de Santiago de Compostela que cursa las materias: Pedagogía Hospitalaria y Diseño y Evaluación de Materiales Didácticos, supervisados por los docentes responsables de las materias. La "Maleta de emergencias pedagógicas" pretende poner a disposición de las aulas hospitalarias, materiales didácticos en soporte impreso, elaborados en su totalidad por estudiantes universitarios y destinados a pacientes pediátricos, profesorado y familias.

Este proyecto tiene una estricta relación con las competencias a trabajar en la titulación de Pedagogía ya que conviene destacar que una de las competencias fundamentales del programa de las materias de Pedagogía Hospitalaria y de Materiales Didácticos está centrada en el diseńo de medios y recursos educativos adaptados y contextualizados a las necesidades educativas en los diferentes contextos y realidades socioeducativas. Igualmente es competencia propia, en la materia de Materiales Didácticos, que el alumnado pueda adquirir estrategias que le permitan diseńar y evaluar los materiales didácticos en diferentes contornos y formatos. En este sentido resulta fundamental que en la asignatura de materiales el alumnado identifique la necesidad de producción de materiales didácticos específicos en el contexto de las aulas hospitalarias. Por otra parte, en el marco de la materia de Pedagogía Hospitalaria resulta fundamental conocer, comprender y analizar de modo integral situaciones educativas en diferentes contextos, como puede ser el hospitalario, siendo capaces de adoptar y aplicar los fundamentos académicos y científicos de la Pedagogía a estos espacios de intervención. También será necesario diagnosticar las necesidades educativas y las posibilidades de desarrollo educativo de la infancia en situación de enfermedad, así como diseñar medios y recursos educativos adaptados y contextualizados en estas necesidades educativas (Universidad de Santiago de Compostela, 2011).

De este modo, siendo conscientes de las potencialidades que ofrece la metodología de trabajo ApS en el contexto hospitalario y tras el análisis de las necesidades y carencias con relación a los recursos y materiales didácticos específicos que permitan el abordaje de ciertos contenidos importantes para el alumnado de un aula hospitalaria decidimos poner en marcha el proyecto: "Maleta de Emergencias Pedagógicas: Materiales Didácticos en aulas hospitalarias".

El objetivo principal del proyecto consiste en diseñar, experimentar, evaluar y crear propuestas de materiales didácticos en distintos formatos adaptados a las necesidades del alumnado del aula hospitalaria.

Se consideran los destinarios directos de la propuesta el alumnado (pacientes pediátricos) y profesorado del aula hospitalaria ya que son los principales destinatarios de los materiales elaborados. Estas propuestas han sido diseñadas con un alto grado de flexibilidad para que puedan ser empleadas con diversidad de pacientes, en relación con su edad, motivo o momento de hospitalización, etc. Igualmente, se ha apostado por el desarrollo de materiales que presentan contenidos especialmente motivadores y terapéuticos y que contribuyan al desarrollo del segui- 
miento del curso académico, que les ayuden a familiarizarse con su nueva situación o simplemente les proporcionen entretenimiento de un modo lúdico y educativo en sus tiempos libres.

Por otra parte, como destinatarios indirectos podríamos mencionar:

El personal sanitario. Las propuestas de materiales permiten a estos profesionales disponer de recursos que ofrecen actividades de las que son copartícipes y también que les sirven de ayuda en el desarrollo de sus actividades. Sobre todo, en lo referente a la sensibilización con diversos procesos médicos o el estado de ánimo de los pacientes pediátricos frente a su situación. A modo de ejemplo, algunos materiales como es el caso de "Afrontando los miedos" y "El dado de las emociones", contribuirán a comprender las intervenciones que impliquen asistencia a un quirófano, o al conocimiento de instrumental médico como por ejemplo las jeringas que suelen ser un elemento estresante y atemorizante para los menores.

Las familias del alumnado. La mayoría de los materiales elaborados buscan la complicidad de las familias en el desarrollo de las propuestas didácticas. Los recursos están pensados para las diferentes fases o momentos del proceso de hospitalización de los pacientes pediátricos. Así, los recursos podrán servir para contribuir a llenar de contenido las esperas en el hospital o bien durante los tiempos de ingreso de los pacientes.

\subsection{FASES DEL DESARROLLO DEL PROYECTO DE APRENDIZAJE-SERVICIO}

Con el propósito de organizar el proceso de desarrollo del proyecto hemos tomado como referencia algunos trabajos centrados en ayudar a estructurar los procesos y fases de elaboración de materiales didácticos.

\subsubsection{FASE INICIAL-CONTEXTUALIZACIÓN}

a) Contacto por parte del profesorado universitario con el aula hospitalaria con el fin de conocer las necesidades específicas de materiales didácticos para trabajar con el perfil de pacientes pediátricos que visitan ese hospital y son destinatarios de la intervención educativa. Es importante destacar que según Puig Rovira et al. la detección de necesidades es un elemento clave en los proyectos de ApS ya que determina el punto de partida al ser un diagnóstico sobre la realidad y sus protagonistas, identificando los elementos susceptibles de mejora del medio en el que se desarrolla, dependiendo la eficacia del servicio en gran medida, de la capacidad de acierto en la definición de estas necesidades iniciales (Puig Rovira et al., 2011).

b) Participación de los docentes universitarios, por invitación de la profesora del aula hospitalaria, en la reunión de inicio de curso desarrollada en el hospital en la que se comparte con el personal sanitario la programación general de los proyectos que se pondrán en marcha desde esta escuela hospitalaria. La realización de este encuentro supuso poder contextualizar la propuesta de nuestro proyecto de ApS en el conjunto de las actividades del aula hospitalaria y también poder transmitir al resto de los profesionales del hospital nuestras contribuciones en la mejora de la calidad de vida del paciente pediátrico. 


\subsubsection{DISEŃO, DESARROLLO, EVALUACIÓN Y EXPERIMENTACIÓN}

a) Sesión Inicial en la que se realiza una reunión con la docente del aula hospitalaria y una vez conocidas las necesidades concretas del tipo de material a elaborar se procede a una reunión conjunta con el alumnado de las materias de Pedagogía Hospitalaria y de Materiales Didácticos con el fin de explicar la propuesta de la experiencia y la iniciativa a desarrollar. Igualmente se detallan las características y las fases a seguir en el proceso de elaboración de los materiales. Se configura la creación de los grupos de trabajo conformados entre 4 y 6 alumnos y alumnas, siendo necesario que en ellos haya, obligatoriamente, alumnado de las dos materias, con el objetivo de no perder la visión interdisciplinar del proyecto y asegurar la presencia tanto de conocimientos teóricos y prácticos adquiridos en la materia de Materiales Didácticos como en la materia de Pedagogía Hospitalaria.

b) Desarrollo: Concepción de las propuestas de materiales, previamente consensuadas con el alumnado y con el personal responsable del aula y el estilo y tipo de material didáctico a desarrollar. Posteriormente detallaremos las propuestas elaboradas. Conviene destacar que en el desarrollo de la iniciativa se ha realizado de modo permanente un registro-diario en el que a través de un drive compartido los miembros coordinadores de la iniciativa anotaban las principales incidencias y aspectos destacables del transcurso del proyecto, tanto en relación con las actividades desarrolladas en el aula, como en relación con los encuentros mantenidos en el hospital y el proceso de experimentación seguido. Igualmente, con el propósito de facilitar la difusión y seguimiento de las propuestas generadas se creó una cuenta de Facebook(https://www.facebook.com/maletadeemerxencias. pedagoxicas.1 ) y otra de Instagram (https://www.instagram.com/maletadeemerxencias/ ), a través de las cuáles se contribuyó de un modo notable a la difusión de la iniciativa y donde pueden observarse los materiales elaborados.

c) Evaluación Inicial: Una vez realizadas las propuestas de materiales por parte de los estudiantes universitarios se desarrolla un primer análisis y valoración de las mismas a través de un trabajo conjuntos entre estos estudiantes, sus docentes y la profesora responsable del aula hospitalaria, con el fin de conocer su viabilidad e interés

d) Experimentación: Incorporadas las revisiones indicadas en la evaluación inicial se procede a su experimentación en el aula hospitalaria con el profesorado y alumnado en el mes de noviembre. Este proceso se realizó de la siguiente manera:

- Encuentro con el alumnado en el aula hospitalaria. Uno de los representantes de cada grupo de trabajo del alumnado universitario llevaba una maqueta del material que tenía diseñado para poner en marcha con los pacientes pediátricos y poder así ver sus potencialidades y debilidades. Los propios estudiantes universitarios en esta experimentación perciben susceptibles mejoras en su material, que posteriormente fueron incorporadas en el diseño final. Entre estos cambios destaca la necesidad de un mayor grado de adaptación a las diversas edades ya que en ocasiones las actividades formuladas eran demasiado fáciles o difíciles para ese rango de edad, cuestiones complicadas de evaluar sin tener la oportunidad de poner en práctica el material con sus potenciales destinatarios finales. 
- Presencia de las familias: es importante destacar que durante esta sesión de experimentación estuvieron presentes las familias del alumnado hospitalizado, quienes se involucraron en la utilización de los diferentes materiales y mostraron su propio punto de vista. Destacaron, el interés del proyecto, ya que hace más ameno el tiempo de espera entre una prueba y otra, y la diversidad de materiales que pudieron experimentar, ya que se ofrecían propuestas que incorporaban el canto, el baile, el teatro, el dibujo, la mímica, etc.

- Reunión con los profesionales del hospital: en el proceso de experimentación, los profesionales del centro destacaron la viabilidad de la iniciativa y algunas mejoras aludían al hecho de aumentar el tamaño de algunos accesorios de los recursos, por ejemplo, pinzas, para que los pacientes de menor edad no pudiesen ingerirlos y la necesidad de que los productos utilizados para la elaboración de los materiales fueran apropiados para esterilizar y desinfectar.

e) Evaluación de los materiales. Teniendo presente que el alumnado que desarrolla la iniciativa forma parte de la materia de Diseño y Evaluación de Materiales Didácticos, en todo momento ha tenido de referencia Guías y Modelos de Evaluación de Materiales que le permitían visualizar aspectos clave en el diseńo tales como: calidad del diseño de los materiales didácticos propuestos, contenidos educativos adaptados, adecuación de las estrategias didácticas planteadas y adecuación de los materiales a la diversidad de pacientes pediátricos

\subsubsection{DIFUSIÓN DE LOS MATERIALES PRODUCIDOS}

Una vez que el material estuvo finalizado se procede a realizar una jornada de presentación del material definitivo en un acto que tuvo lugar en el propio complejo hospitalario con la participación del alumnado implicado en el proyecto, los responsables de las materias y la responsable del aula hospitalaria. En esta sesión, cada grupo realiza una presentación del material final explicando las características y funcionalidad de este.

En el acto de presentación de la actividad estuvo presente la directora de procesos asistenciales del hospital, la maestra responsable del aula hospitalaria, el alumnado y profesorado de las materias, así como diferentes profesionales del hospital (personal médico y de enfermería). Así mismo, también asistió como invitado el jefe territorial de Educación de la provincia. En este acto, la docente del aula hospitalaria definió el producto final como "una maleta que se puede trasladar de habitación en habitación, al igual que cuando los niños y niñas dejan de estar hospitalizados y va una profe a sus casas durante tiempo" e incidió en la importancia de la iniciativa para que "el nińo conozca sus derechos y reconozca sus miedos". (Véase algunas notas de prensa en las cuáles se recogen diversas opiniones de los participantes en el proyecto, https://cutt.ly/ui6G9Q, https://cutt.ly/Xi61gU, https://cutt.ly/ni60km, https://www.usc.gal/ es/node/481).

\section{LAS EVIDENCIAS Y LOS RESULTADOS DEL PROYECTO DE APRENDIZAJE-SERVICIO}

\subsection{MATERIALES ELABORADOS}

Se trata de ocho materiales los que componen la "Maleta de emergencias pedagógicas" dirigidos a pacientes pediátricos, todos ellos están adaptados a las diferentes edades de los pacientes pediátricos (entre los 3 y los 14 años), que fueron construidos con materiales que se 
pueden desinfectar y cuentan con guías e instrucciones para que puedan ser empleados de manera autónoma por el alumnado o también en equipos orientados o no por la docente.

A continuación, se exponen de un modo muy resumido las características de cada uno de los materiales elaborados dentro del proyecto global. Conviene destacar que han tenido como referencia los siguientes temas:

- Identificación y tratamiento de las emociones que experimenta la infancia hospitalizada.

- $\quad$ Espacios y profesiones del hospital, desde una perspectiva de género.

- $\quad$ Enfermedades y dolencias más comunes en la infancia trabajadas en ese centro.

- La música: composición y grabación de dos canciones para trabajar el miedo a las inyecciones y jeringas y al quirófano, así como una propuesta de musicoterapia.

- $\quad$ El contorno de Ferrol: mediante un juego sobre hombres y mujeres ferrolanas de reconocido prestigio por su actividad.

De un modo sintético, podemos ver en el cuadro 1 los materiales elaborados.

\section{Cuadro 1}

Características materiales elaborados

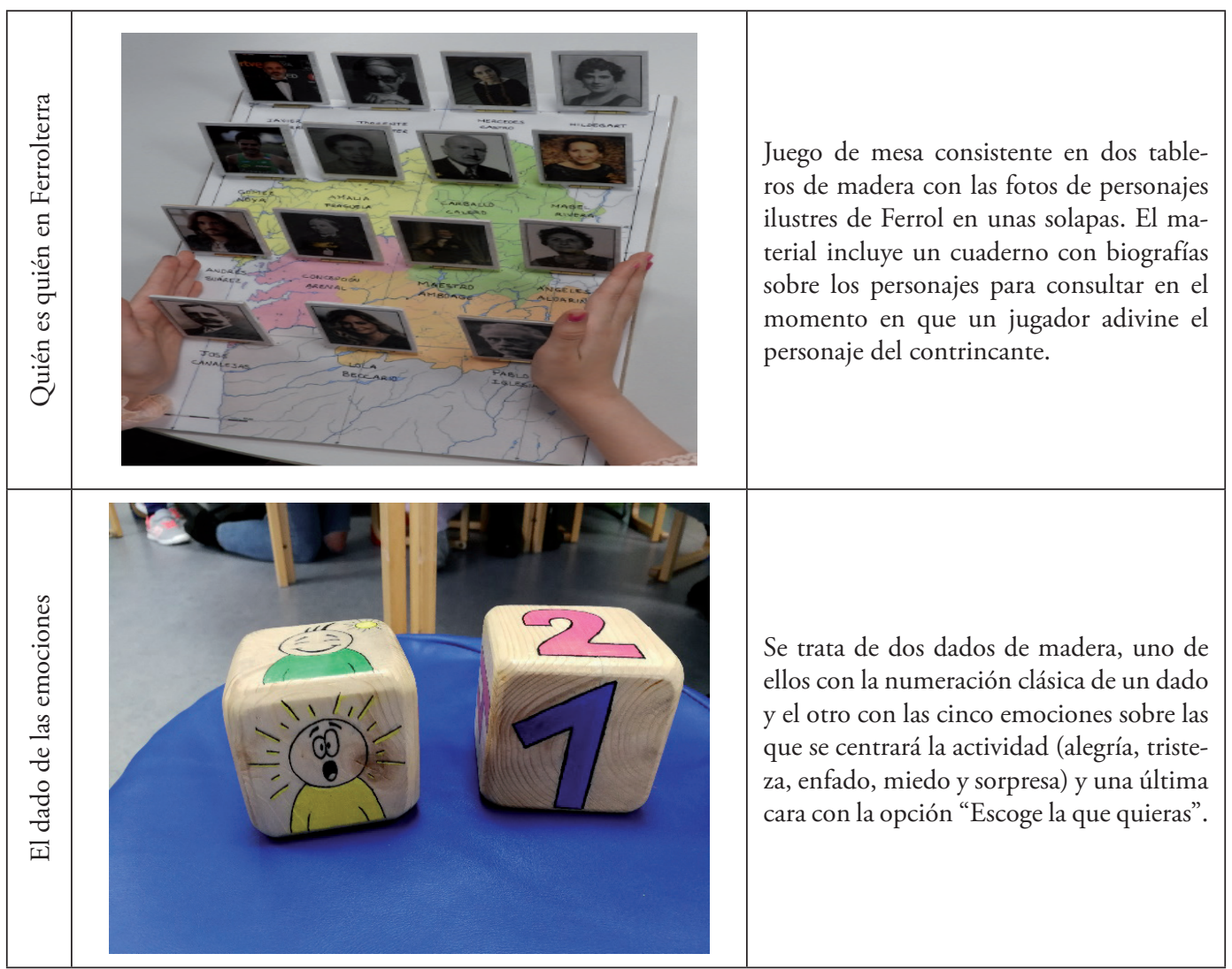




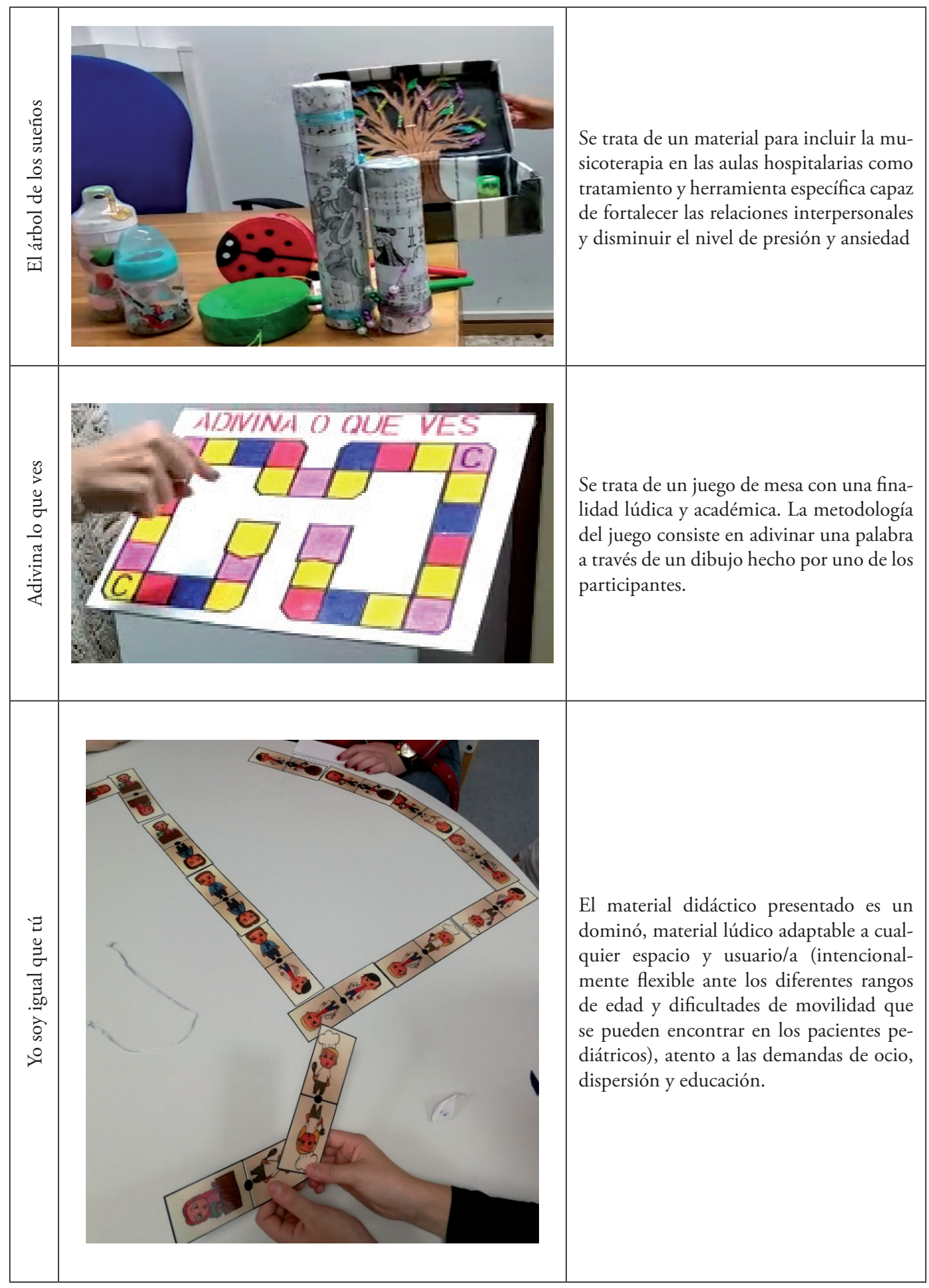




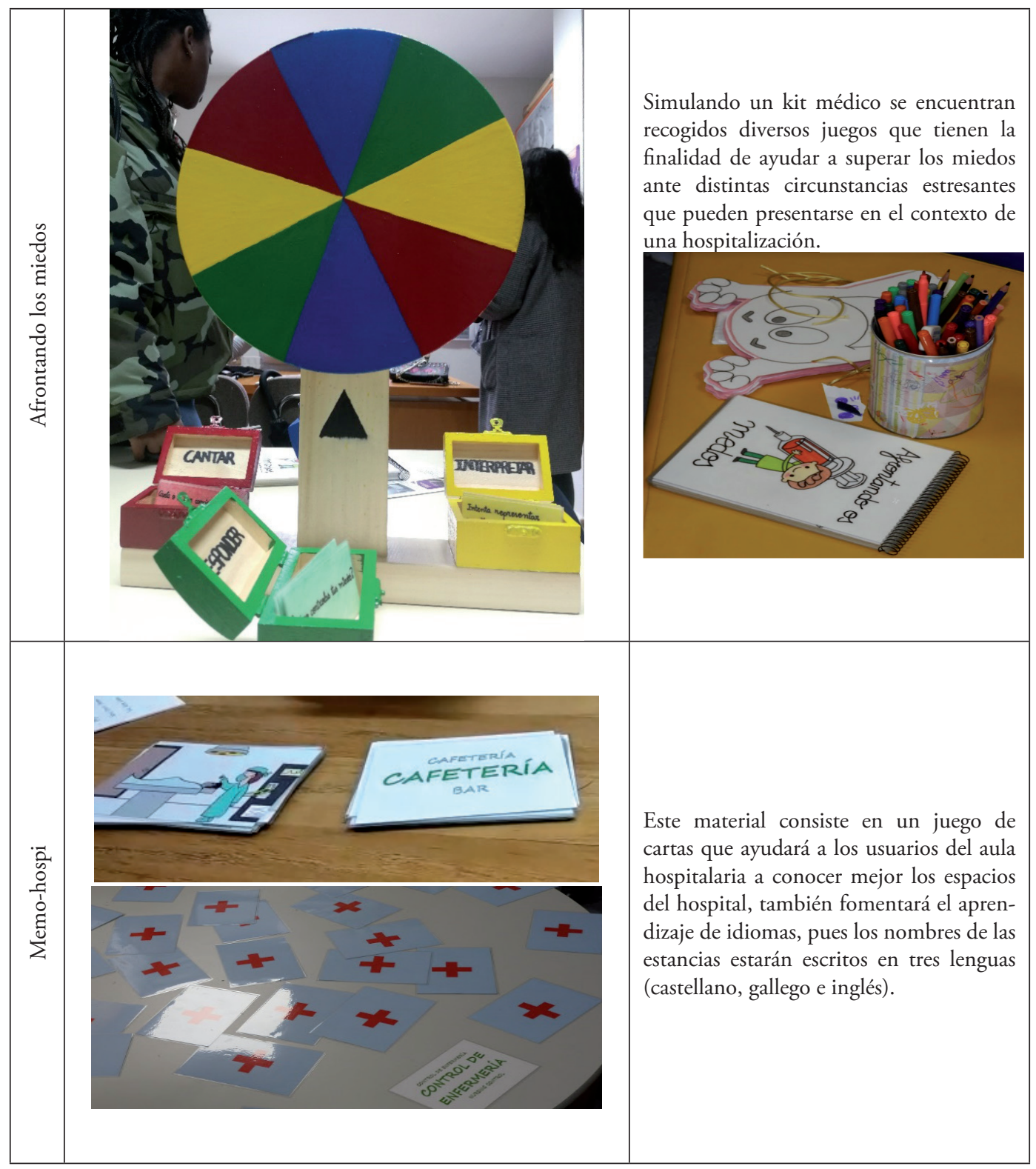




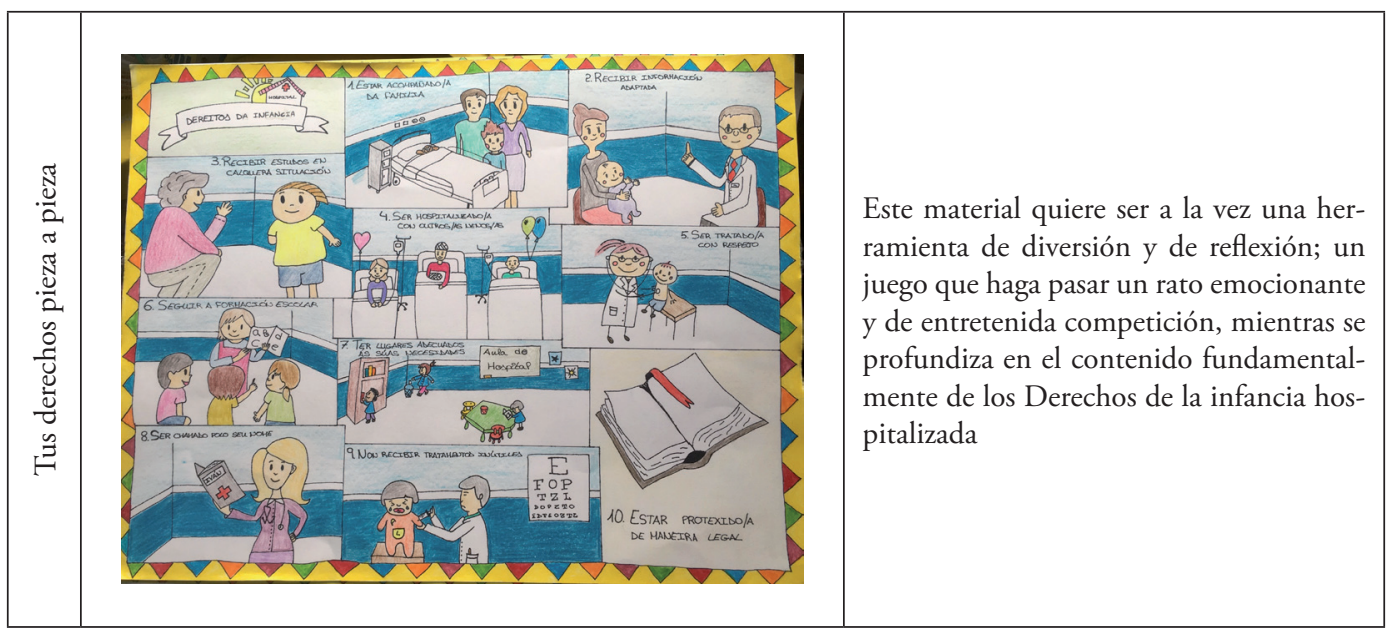

\subsection{APRENDIZAJES Y VALORACIONES DEL ALUMNADO}

Una vez finalizado el proyecto y acompańando cada uno de los materiales elaborados, se le pidió al alumnado universitario un informe sobre cada uno de los materiales con el propósito de evaluar el conjunto del proyecto desarrollado.

Además, en la evaluación final de ambas materias se evidenció como la participación del alumnado universitario en el proyecto de Aprendiza-Servicio contribuyó a la adquisición de aprendizajes como los siguientes:

- El diseño de medios y recursos educativos adaptados y contextualizados a las necesidades educativas del contexto de las escuelas hospitalarias.

- La detección de necesidades específicas en relación con los materiales didácticos que se presentan en un aula hospitalaria.

- El diseño y evaluación de materiales didácticos en diferentes formatos.

- La elaboración de propuestas de intervención que favorecen el seguimiento de los procesos de escolarización que se desarrollan en el contexto hospitalario.

- El conocimiento, la comprensión y el análisis integral de situaciones educativas en el hospital.

Además de evaluar los aprendizajes adquiridos también contamos con la valoración y opinión del alumnado universitario implicado, así como la opinión de los diferentes profesionales e instituciones que han participado en el desarrollo de la iniciativa para la evaluación del proyecto.

Con el propósito de conocer la opinión de los cincuenta alumnos y alumnas que han participado en el diseño de los materiales, se aplicó un cuestionario de nueve preguntas en el que se combinaban ítems de respuesta cerrada con otros de respuesta abierta, con el objetivo de descubrir los principales aprendizajes extraídos por el alumnado, los aspectos más relevantes de la experiencia para su posterior desempeño profesional, la satisfacción, implicación y valoración del proyecto, así como cambios y sugestiones de mejora. 
En cuanto a los principales aprendizajes, el alumnado destaca "poder aplicar los conocimientos teóricos sobre la realización de proyectos y diseńo de materiales adquiridos desde el $1^{\circ}$ curso", "aprendizajes personales como son valores éticos, sociales, de solidaridad" y el desarrollo de una de las competencias que debiera estar presente en todas las materias universitarias, el espíritu crítico: "crear una mentalidad crítica en cuanto a los materiales didácticos y la importancia de los mismos". Así mismo, resaltan la oportunidad de trabajar en un nuevo contexto con dinámicas diferentes a las habituales, tal y como se refleja en la siguiente afirmación: "una mejor aproximación a la realidad hospitalaria desde la perspectiva pedagógica, nuevas estrategias de intervención educativa en situaciones diferentes a las estudiadas previamente, nuevos contextos y nuevas metodologías de actuación socioeducativa además de aumentar y mejorar capacidades y competencias en términos de habilidades sociales, trabajo en equipo, comunicación, etc."

Estableciendo una relación entre esta experiencia de ApS y su posterior desempeño profesional, todas las valoraciones coinciden en la oportunidad brindada de conocer o familiarizarse con un potencial contexto de trabajo como son las aulas hospitalarias y la posibilidad de poner en práctica competencias necesarias para desarrollar esta labor como "el trabajo en equipo o la capacidad de tolerancia a la frustración”. En palabras del alumnado, afirman que "conocen mejor la labor que las pedagogas realizan en los hospitales, en general, y en las aulas hospitalarias, en concreto" y que tuvieron la posibilidad de "aproximarse más directa y transparentemente a la realidad profesional como pedagoga”.

En relación con aquellas cuestiones que cambiarían o mejorarían del desarrollo del proyecto, hacen hincapié en la posibilidad de aumentar los períodos temporales dedicados en ambas materias a la realización de este proyecto de ApS. Así mismo, recogen que sería necesario elaborar otros materiales didácticos como cuentos interactivos en los que los pacientes pediátricos fuesen también personajes, recursos para atender la diversidad funcional (deficiencia visual, autismo, trastornos neurológicos) o materiales para implicar las familias en el aula hospitalaria.

El alumnado participante en esta experiencia considera que los destinatarios y profesionales del hospital pudieron aprender de sus ideas como futuros pedagogos y pedagogas y que se estableció "una comunicación interprofesional que contribuyó al desarrollo de la actividad hospitalaria, desde la colaboración de procesos de humanización hasta la mejora del labor educativo en un aula hospitalaria”, obteniendo por tanto beneficio ambas partes, tanto alumnado como profesional sanitario y pacientes pediátricos, tal y como recoge una de las alumnas "supuso gratitud al sentir que las necesidades del hospital de Ferrol son contempladas y que de modo solidario se colaboró con ellos para mejorar la situación. La oportunidad de aprender unos de los otros a la vez".

\subsection{EL PAPEL DE LA UNIVERSIDAD}

Uno de los aspectos que hemos reseńado como fundamental en este proyecto ha sido el hecho de que la universidad a través de sus investigadores e investigadoras se haya visto involucrada desde un primer momento en la ejecución del proyecto. Entre otros aspectos, es conveniente tener presente en qué medida los propios miembros de la universidad y de las distintas instituciones se han enriquecido al formar parte de una iniciativa de ApS. Desde nuestra perspectiva, y como docentes universitarios, consideramos que ha resultado sumamente intere- 
sante poder realizar una aplicación de los planteamientos teóricos sobre los procesos de elaboración de materiales que manejamos en el contexto universitario a la construcción de materiales didácticos en las Aulas Hospitalarias y, por otra parte, el poder adentrarse en las necesidades, problemas, rutinas diarias y planteamientos educativos en el marco del contexto hospitalario.

\subsection{EL PAPEL DEL HOSPITAL}

El hospital, -que ha sido copartícipe en todo momento en las fases del desarrollo del proyecto- ha encontrado en el desarrollo de este una oportunidad para contar con una propuesta de materiales didácticos que le ha permitido atender las necesidades de los docentes del aula hospitalaria y personal sanitario relacionadas con la ocupación del ocio del alumnado, conocimiento de las actividades y estructura del complejo hospitalario, "sensibilización" hacia determinados temas como la entrada en un quirófano, la aplicación de inyecciones, etc. En este sentido, todo el personal sanitario presente en las sesiones de experimentación de los materiales como en el acto de entrega mostró su agradecimiento y el interés de darle continuidad a la experiencia. Igualmente, el desarrollo de esta iniciativa posibilitó que el hospital sea copartícipe de actividades relacionadas con el conocimiento del entorno y la normalización lingüística ya que todos los materiales elaborados están en lengua gallega.

\section{VALORACIONES FINALES}

Finalizar este primer período del proyecto nos ha permitido experimentar como un programa puede contribuir a cubrir necesidades básicas en la pedagogía hospitalaria y la humanización de la atención educativa que se aplica dentro de los contextos sanitarios a la vez que permite mejorar mucho los conocimientos y habilidades de alumnado universitario que en su futuro profesional puede estar trabajando en este campo. Teniendo presente que cuando un menor está hospitalizado o en situación de enfermedad, el modo en que debe de trabajarse con ese paciente pediátrico y esa familia que lo acompaña dentro del contexto hospitalario, debe de ser siempre con una óptica de recuperar o restaurar su salud desde una perspectiva de atención integral. Apostando, por tanto, por procesos asistenciales en los que, será imprescindible, mejorar la humanización, en convivencia con la tecnificación tanto en el campo de la medicina, como en el campo educativo y social (Molina, 2020). Lo cual implicará, necesariamente, un trabajo en equipo e interdisciplinar de los agentes profesionales implicados en el proceso de recuperación del menor dentro de su unidad familiar.

En este sentido, las últimas investigaciones y publicaciones en el campo apuestan por atenciones integrales en las que se tenga en cuenta la valoración y satisfacción de los pacientes. Siendo así, "la pedagogía hospitalaria es uno de los componentes de la atención y un elemento de humanización de la asistencia, puesto que responde a las necesidades educativas, articulada con la atención sanitaria, psicológica y social” (Molina, 2020, p. 73). Conseguir esto que Molina le plantea a la Pedagogía Hospitalaria, pasa por la necesidad de apostar por una mejor formación de los profesionales del campo, así como mejorar algunos de los puntos débiles de esta disciplina. En esa línea, lo que se apunta en investigaciones recientes es la importancia de la mejora de los recursos materiales y humanos, algo que valoran tanto desde el colectivo del personal sanitario como desde el colectivo del personal docente que trabaja en las aulas hospitalarias (Teijeiro, 2019). 
Teniendo en cuenta lo expuesto hasta ese momento, valoramos positivamente la aportación de esta experiencia de ApS, ya que el alumnado tuvo la oportunidad de elaborar propuestas de materiales didácticos que respondan a necesidades concretas en el marco de las aulas hospitalarias a la vez que pudieron formarse y experimentar retos que su futuro profesional les depara, bien en el diseño y la elaboración de materiales didácticos que supongan un recursos imprescindible para responder a la diversidad del alumnado, bien desarrollando y poniendo en práctica competencias que necesitarán en un futuro próximo si desempeńan su labor profesional en un contexto como el hospitalario.

Para el equipo coordinador del proyecto, también supuso un importante espacio de reflexión y de debate, favorecido por la constitución de un equipo interdisciplinar de trabajo con formaciones y planteamientos diferentes, pero con un interés común: el de contribuir a que el alumnado de las aulas hospitalarias dispusiese de propuestas de materiales adaptadas a sus necesidades concretas.

En medio de este espacio interactivo reflexionamos sobre el papel de los materiales, compartiendo conocimiento, ideas, poniendo en la mesa todos nuestros preconceptos sobre la función de los materiales y sobre las características del sistema hospitalario, etc. En definitiva, el proceso de diseńo, desarrollo y evaluación del proyecto supuso un importante aprendizaje para todo el equipo implicado. Incrementando los conocimientos sobre el contexto de elaboración de materiales en las aulas hospitalarias y, por otra, desarrollando interesantes procesos de reflexión acerca de los modos de elaboración de materiales.

Para finalizar, también conviene hacer referencia a la necesidad y viabilidad de afrontar nuevos retos en futuras ediciones de este proyecto, entre los que consideramos importante destacar los siguientes:

- El principal impulso vendría dado porque las autoridades asuman la importancia que poseen las iniciativas de estas características y tomen conciencia de proveer fondos, planificación y estrategias para su desarrollo de un modo constante y organizado.

- Sería interesante contar con nuevos profesionales que ayuden en el desarrollo de materiales de calidad tal es el caso, por ejemplo, de diseñadores profesionales que permitieran una mejora cualitativa de las ilustraciones de los recursos.

- Un nuevo interés en este proyecto vendría dado por la incorporación de material interactivo, y por la inclusión de otras tecnologías en la propuesta ya que podrían contribuir en una infinidad de aspectos en el desarrollo de proyectos educativos en las aulas hospitalarias.

- Igualmente, en el desarrollo del proyecto se pone de relieve la necesidad de que los materiales didácticos habitualmente empleados en el contexto escolar, deberían reflejar iniciativas que permitan trabajar con las experiencias del menor hospitalizado, de tal manera que la realidad del paciente pediátrico no suponga un hecho aislado y con sólo implicaciones educativas para el propio sujeto, sino también para sus compañeros y compañeras de aula.

- Sería conveniente que el desarrollo de este tipo de propuestas se extendiera a otros hospitales. 


\section{REFERENCIAS}

Grau, C, \& Ortiz., M. C. (2001). La pedagogía hospitalaria en el marco de una educación inclusiva. Aljibe.

Guerra, J., \& Revuelta, F. I. (2015). Videojuegos precursores de emociones positivas: propuesta metodológica con Minecraft en el aula hospitalaria. International Journal of Educational Research and Innovation, 3, 105-120.

Guilló, J. (2006). La Convención sobre los derechos del Niño. Derechos y Necesidades de la Infancia. In C. Escobar, G. Sánchez, \& T. Andrés (Eds.), Trabajo Social, Familia y Mediación. Necesidades Sociales en la Infancia y Derechos del Niño. Ediciones Universidad Salamanca.

Latorre, M. J., \& Blanco, F. J. (2010). Función profesional del pedagogo en centros hospitalarios como ámbitos educativos excepcionales. Educación XX1, 13(2), 95-116.

Lizasoáin, O., \& Polaino-Lorente, A. (1998). Evaluación de la modificación del autoconcepto infantil como consecuencia de la hospitalización. Acta Pediátrica Española, 46(1), 13-18.

Molina Garuz, M. C. (2020). Pedagogía Hospitalaria. Claves teóricas y enfoques para la práctica. Grao.

Ortiz, M. C. (2001). Aproximación a la Pedagogía Hospitalaria. In M. A. Verdugo, \& F. Borja Jordán (coords.), Apoyos, autodeterminación y calidad de vida: Actas de las IV Jornadas Cientificas de Investigación sobre Personas con Discapacidad (pp. 633-642). Amarú.

Ottaviano, M., \& González, J. (2015). Estrategias didácticas para los docentes hospitalarios y domiciliarios. In C. S. Gónzalez, \& V. Violant (Eds.), Uso de las TIC para la atención educativa, hospitalaria y domiciliaria (pp. 67-101). Mc Graw Hill Education.

Puig Rovira, J. M., Battle, R., Bosch, C., \& Palos, J. (2006). Aprenentatge servei. Educar per a la ciutadanía. Octaedro.

Puig Rovira, J. M., Gijón Casares, M., Martín García, X., \& Rubio Serrano, L. (2011). Aprendizajeservicio y Educación para la Ciudadanía. Revista de Educación, número extraordinario, 45-67. http://www.revistaeducacion.educacion.es/re2011/re2011_03.pdf

Rodríguez Gallego, M. R. (2014). El Aprendizaje-Servicio como estrategia metodológica en la Universidad. Revista Complutense de Educación, 25(1), 95-113. https://revistas.ucm.es/index.php/ RCED/article/view/41157

Teijeiro Bóo, Y. (2019). Experiencias y programas educativos para la infancia en contexto hospitalarios: un estudio pedagógico-social acerca de sus realidades y perspectivas de futuro (Tesis doctoral inédita, Universidad de Santiago de Compostela). Fundación Dialnet. https://dialnet.unirioja.es/servlet/ tesis? codigo $=234008$

Universidad de Santiago de Compostela. (2011). Memoria para la verificación del título de Grado en Pedagogía. http://www.usc.es/export9/sites/webinstitucional/gl/servizos/sxopra/memorias_graos_ USC/Pedagoxxa.pdf

Recebido em: 30/06/2021

Reformulado em: 30/08/2021

Aprovado em: 12/09/2021 